
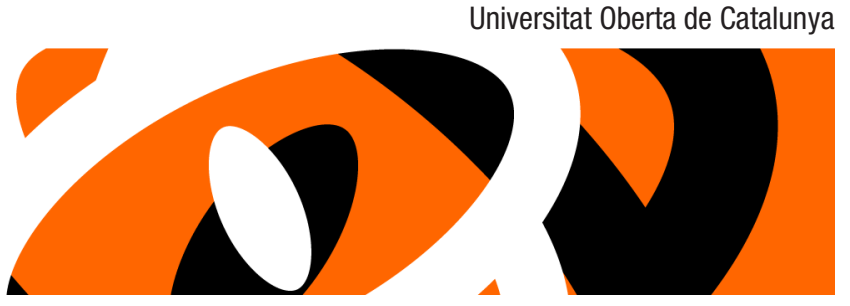

http://artnodes.uoc.edu

\title{
Apocalipsis media. Disección, regeneración y reparación: agentes para la fabricación de sistemas zombis lúdicos-tecnocráticos
}

\section{Marina Pastor \\ Moisés Mañas \\ Universitat Politècnica de València}

Fecha de presentación: febrero de 2018

Fecha de aceptación: abril de 2018

Fecha de publicación: junio de 2018

\section{Cita recomendada}

Pastor, Marina; Mañas, Moisés. 2018. «Apocalipsis media. Disección, regeneración y reparación: agentes para la fabricación de sistemas zombis lúdicos- tecnocráticos». En: Pau Alsina, Ana Rodríguez y Vanina Y. Hofman (coords.). «Arqueología de los medios». Artnodes. N. ${ }^{\circ} 21$ : 54-64. UOC [Fecha de consulta: dd/mm/aa]. http://dx.doi.org/10.7238/a.v0i21.3178.

Este artículo está sujeto -si no se indica lo contrario- a una licencia de Reconocimiento 3.0 España de Creative Commons. Puede copiarlos, distribuirlos, comunicarlos públicamente, hacer obras derivadas y usos comerciales siempre que reconozca los créditos de las obras (autoría, nombre de la revista, institución editora) de la manera especificada por los autores o por la revista. La licencia completa se puede consultar en http://creativecommons.org/licenses/by/3.0/es/deed.es. 


\section{artnodes}

http://artnodes.uoc.edu

Apocalipsis media

\section{Resumen}

Partiendo de la idea de juego como agente de relación, planteamos en este texto el estudio e interpretación de la relación y resoluciones que subyacen entre términos como los de disección, regeneración y reparación, que operan a manera de elementos partícipes en la fabricación de sistemas interactivos. También abordamos la relación de estos con la obsolescencia tecnológica como un modelo para la construcción de escenarios idóneos en la creación de sistemas lúdicos atemporales, que utilizan el concepto tecnocrático de resolución de problemas en pro de conseguir de manera lúdica, y desde la acción social, la resurrección de valores para la mediación y la creación artística visual.

De este modo, si la presencia del juego permite rehacer, cuestionar e interpretar la realidad reelaborándola en el pensamiento estético, y aproximándola a la experiencia social cotidiana compartida, la zombificación es un modo de hacer frente a la obsolescencia planificada desde una micropolítica lúdica, para incidir en una teoría de los medios de carácter práctico, ético y estético, conformando una cultura de los medios más ecológica. Proponemos que la reutilización lúdica es una dinámica importante en la cultura contemporánea, a la que añade una dimensión reflexiva y crítica, que es especialmente relevante dentro del contexto de los residuos electrónicos. En ella, la arqueología de los medios, convertida en anarqueología, puede funcionar como una metodología artística que se inserta en la tradición de la apropiación, el collage, la remezcla de medios materiales y conceptos. Se trata de excavar en narrativas y medios materiales de tal modo que se produzca en una nueva economía política de los medios.

\section{Palabras clave}

medios zombis, arqueología de los medios, agenciamiento, anarqueología, arte industrial

\section{Media-apocalypse. Dissection, regeneration, and repair: agents for the development of technocratic-ludic zombie systems}

\section{Summary}

In this text, we explore the study and interpretation of the relationship existing between and the resolution underlying terms such as dissection, regeneration and repair based on the idea of the game as a relational agent. These terms operate as elements that participant in the development of interactive systems. We also explore their relationship with technological obsolescence as a model for the construction of ideal settings in the creation of atemporal recreational systems, which use the technocratic concept of problem resolution to achieve the resurgence of values for mediation and visual artistic creation in a recreational way based on social action.

Thus, if the presence of the game facilitates the re-invention, questioning and interpretation of reality, re-developing it within aesthetic thought, as well as bringing it closer to the shared daily social experience, zombification is a way of approaching planned obsolescence from a recreational micro-political perspective. Its purpose is to contribute to media theory that is practical, ethical, aesthetic and creates a more ecological media culture. We propose that recreational re-use is an important dynamic in contemporary culture, to which a critical and reflective dimension is added; this is especially relevant within the context of electronic refuse. Within this dynamic, media archaeology (transformed into an-archaeology) is able to function as an artistic methodology that makes it place within the tradition of appropriation, the collage, the re-mixing of concepts and material media. This text excavates narratives and material media such to produce a new political economy of media.

\section{Keywords}

zombie media, media archaeology, agency, anarcheology, industrial art 


\section{artnodes}

\section{Introducción}

El 1 de marzo de 2017, el senador valenciano por el partido político de Compromís Carles Mulet interrogaba, en una sesión de control parlamentario al gobierno, acerca de los protocolos específicos establecidos en la nación española ante la posibilidad de un apocalipsis zombi. Aunque en realidad Mulet pretendía poner en evidencia los impedimentos que comúnmente el poder ejecutivo desarrolla al resolver cualquier cuestión concreta, apelando a la legislación ya conocida de modo genérico y poco preciso, la pregunta parece absolutamente pertinente si tenemos en cuenta que una de las condiciones clave que desarrollan las sociedades occidentales contemporáneas es el miedo y las correspondientes teorías de carácter apocalíptico.

Hoy, «el miedo es el mensaje» (Gil Calvo 2003), y se han convertido en un principio generalizado, común y cotidiano, las afirmaciones de Beck sobre las sociedades del riesgo, en las que exponía el origen de nuestros miedos en la lógica de desarrollo de la modernidad y, muy particularmente, en la evolución incontrolable de la ciencia y la tecnología, apuntando el paso de una sociedad de clases a una sociedad de riesgos. Con el inicio del milenio, los riesgos se transforman para hacerse globales: «Riesgo es el enfoque moderno de la previsión y control de las consecuencias futuras de la acción humana, las diversas consecuencias no deseadas de la modernización radicalizada. Es un intento (institucionalizado) de colonizar el futuro, un mapa cognitivo. Toda sociedad, por supuesto, ha experimentado peligros. Pero el régimen de riesgo es una función de un orden nuevo: no es nacional, sino global» (Beck 2002, 5).

Según Gil Calvo (Gil Calvo 2003), el principio de acuerdo con el cual se desenvuelve el miedo contemporáneo global responde a la lógica de desarrollo de las tecnologías de la información y la comunicación. Las razones son las siguientes:

1. Las tecnologías de la información y la comunicación incrementan las interacciones y las dependencias y, con ello, aumenta la sensación de que un mismo evento puede afectar a más personas.

2. La reciente crisis de los estados-nación, incardinada bien a través de asociaciones económicas y/o políticas entre estados, que desemboca en el «estado red» (Castells 2006), bien reivindicando principios de independencia ante las naciones de pertenencia, acrecienta la sensación de que no hay control ni previsión institucional. Los dos motivos fundamentales son, para Castells, la globalización de la economía y su organización en relación con redes de interacción de información, y la incapacidad del Estado de lograr una identificación con las necesidades de la sociedad civil. La crisis desemboca, en este punto, en una crisis de legitimidad (Castells 2006, 393).
3. El incremento cuantitativo de las formas de interacción hace imposible el cálculo de las consecuencias de cualquier acción, ya sea individual 0 colectiva.

4. En la era pre-internet, la realidad se percibía de una manera natural y racional; hoy emerge de la interacción, es decir, como una consecuencia de la agregación colectiva de microcomportamientos (Gil Calvo 2003, 165), como su consecuencia imprevista o no intencionada.

\section{Miedo y Consumo S. L.}

Con todo, la globalización ha conferido extraordinarias capacidades a los medios de comunicación para transmitir información. Los medios crean climas de opinión, pueden transmitirnos la sensación de estar viviendo un riesgo de manera próxima cuando en realidad su correlato material está muy alejado del contexto vital. Los media, a su vez, crean sus propios riesgos y tienden a generar un estado de alerta colectivo no ajustado al contexto real en el que vivimos puesto que ya no podemos reconciliar siempre la información con los criterios de contraste y comprobación, con los criterios de verificación, al menos en tiempo real. ${ }^{1}$ Por último, no podemos olvidar que la inseguridad y la incertidumbre son grandes argumentos para estimular la idea de que el control es afortunado y podemos sentirnos felices si estamos controlados.

La misma clase de operaciones se producen hoy con el consumo, pautado por la obsolescencia programada y basado en la generación de necesidades secundarias. Cada cual se siente feliz por desechar sus máquinas buscando el último modelo tecnológico que necesita por razones no estrictamente lógicas. Si unificamos las pautas de miedo, riesgo e incertidumbre y consumo, encontramos el modo en el que la sociedad construye sus imaginarios, delinea sus deseos, modifica sus prácticas cotidianas y construye espacios simulados y de simulación. Juntas conforman un territorio (poder) y un paisaje: el régimen escópico del control que Deleuze teorizó en su Post scriptum (Deleuze 1999, 277).

\section{Apocalipsis: zombis o creativos}

Nuestro imaginario tiende a ser cada vez más el del Apocalipsis, como atestiguan fenómenos mediáticos y toda la iconografía mediática, artística y lúdica del terror, repleta de vampiros, hombres lobo y zombis. En nuestras fantasías apocalípticas se delinea una carencia fundamental: algo escasea, algo se rompe, todo deja de funcionar...

1. Como ejemplo reciente, tenemos lo acontecido en Hawái el 15 de enero de 2018, en el que toda la población recibió, a las 8:08 de la mañana, la siguiente alerta de emergencias: «Alerta de misil balístico en dirección a Hawái. Busque refugio de inmediato. Esto no es un simulacro». El mensaje fue enviado por el servicio de emergencias estatal por el error de un empleado. 


\section{artnodes}

http://artnodes.uoc.edu

Apocalipsis media

Se produce el fin del mundo, pero a él sobreviven siempre unos desvalidos sujetos que tendrán que asumir de nuevo el control del mismo. La figura del apocalipsis, no obstante, se nutre de formas diversas: el 7 de mayo de 2010 se produce lo que Ted Kaufman, senador demócrata, denominó «la batalla de los algoritmos», una caída repentina en el índice Dow Jones generada por un error en los algoritmos que controlan los ordenadores, que fueron contaminados por una serie de operaciones incapaces de detenerse en su high frequency trading (Pozzi 2010). Lo que este ejemplo pone en evidencia es un supuesto potencial atribuido a la informática para alterar el mercado y crear el caos, como si el mercado fuera una cosa y los ordenadores otra, como si los superordenadores no fueran la génesis del funcionamiento y las dinámicas de los mercados contemporáneos. Con frecuencia, se dota a la máquina del protagonismo, y se limita el papel del fallo humano para someternos a una sensación creciente de fragilidad y descontrol del mundo, para hacernos aceptar que son necesarios controles suplementarios si queremos que no todo escape del nuestro. No obstante, para muchos de nosotros, lo que esto pone en evidencia es que, cuando las cosas se rompen, cuando fallan los algoritmos, cuando algo deja de funcionar, es mucho más interesante. En el caso de la mediación tecnológica, lo que se nos revela es la autenticidad de lo real, ahora no mediado, propiciando la toma de conciencia de la mediación tecnológica. ${ }^{2}$ No obstante, el fallo también tiene consecuencias no solo del lado de la realidad, sino también en relación con el polo subjetivo, en el que, de manera metafórica y simplificada, se manifiestan tres tipos de humanidad. Por una parte, se encuentran los tecnócratas: son aquellos que comunican a los ordenadores qué es lo que deben hacer; por otra, los zombis: aquellos que hacen lo que dicen los ordenadores. Por último, los creativos, usualmente también llamados freaks o geeks, etc., que con frecuencia se mueven entre los agujeros de los dos extremos anteriores, apasionados por los errores y el descrédito que estos inducen tanto en la subjetividad como en la realidad, y que saben aprovechar lúdicamente los desgarrones de su tejido.

Podemos aludir en este punto a Ralph Koster, un desarrollador de videojuegos que, en la actualidad, se dedica a hacer de consultor, dar conferencias y publicar ensayos como $A$ Theory of Fun. Koster, que fue conocido inicialmente por diseñar la gameplay de Star Wars Galaxies y por ser el primer diseñador de un juego multijugador en línea, relata cómo, debido a la presión de los grandes estudios, la visión que él estimó que debía tener el juego nunca llegó a completarse, lo que condujo al primer gran fracaso de la historia de los MMORPG. En su página web reflexiona acerca de cómo los derechos, individuales e inalienables, que definen lo que es una persona no son otorgados por nadie, sino que decidimos dárnoslos a nosotros mismos. A partir de esta idea establece que los administradores de videojuegos multijugador en línea, con frecuencia, entienden sus áreas como espacios privados en relación con los cuales pueden tomar decisiones arbitrarias opuestas al propio concepto de derecho, que debe extenderse al espacio virtual. Para ello propone una reformulación de los derechos de los jugadores, expresados como derechos de los avatares, de acuerdo con el cual reconoce un papel creativo a los propios jugadores, cuyos derechos deben ser respetados. Esta idea de creatividad es especialmente aludida en el tercero de los derechos reconocidos, que se concreta del siguiente modo:

«El principio de toda soberanía en un espacio virtual reside en el hecho inalterable de que en algún lugar hay una persona que controla el hardware en el que se está ejecutando dicho espacio virtual y el software con el que se creó, y la base de datos que constituye su existencia. Sin embargo, los habitantes de ese espacio virtual tienen el derecho de conocer y exigir el cumplimiento de las normas por las que esta persona utiliza ese poder sobre la comunidad. La autoridad debe proceder de la comunidad: por lo tanto, una comunidad que no conoce las normas por las que los administradores utilizan su poder es una comunidad que permite que sus administradores no tengan normas, y es una comunidad que manifiesta complicidades con la tiranía» (Koster 2000).

Más allá de la pertenencia de Koster al universo empresarial mediático, recogida en su propia trayectoria personal, nos interesa rescatar la modulación de la actitud creativa que expresan sus derechos y cómo esta despliega la negación a la colaboración con cualquier clase de autocracia. Entendemos que los jugadores creativos son los que se niegan al colaboracionismo con cualquier clase de despotismo. Esta actitud es extensible a la creatividad propia de la producción artística y se modula en trabajos como el de Melissa Barron, que se concreta en un entorno de negación de la colaboración con los media de forma tradicional, en particular Untitled (Barron 2010). En esta serie, Melissa captura los defectos gráficos que surgen de manera emergente y espontáneamente en la pantalla de un viejo computador Apple II mientras intenta jugar a un videojuego de Activision. Estas imágenes capturadas, muertas, supuestamente erráticas y ejemplo de representación glitch, son impresas en un telar jacquard TC-1 computerizado generando una transformación en la fabricación de lo digital a lo analógico, produciendo un elemento moribundo tecnológicamente fruto de un proceso industrial errático que define esta relación entre medios como una relación zombi, una representación de un cadáver andante.

El origen metodológico del consentimiento hacia lo tiránico se encuentra en la diferencia epistemológica entre los conocimientos de caja transparente o de caja negra, para responder al orden económico mundial y a la situación de dependencia de las grandes industrias

2. El ejemplo cotidiano puede ser un ascensor estropeado, que revela la altura real a la que vivo cuando debo alcanzarla subiendo por las escaleras 


\section{artnodes}

económicas. Cada vez nuestra cultura mediática se desarrolla más en la oscuridad, y lo que zombifica a los usuarios está relacionado con el concepto de puntualización desarrollado por Latour (Latour 2001, 220-221). Para explicar nuestra lectura de este, podemos usar el ejemplo de un coche. La mayoría de los usuarios zombificados conciben el automóvil como un objeto único (eso es estrictamente la puntualización), hasta que se estropea. Entonces observan que es una colección de componentes conectados y piensan que existe un fallo en alguno de ellos. El desconocimiento de dichos componentes y sus conexiones, la formación de cajas negras, depende de los intereses comerciales. En relación con la tecnología, los usuarios suelen desconocer la tríada sobre la que esta se asienta: tanto el software como el hardware, además de las teorías sobre las que estos se asientan. Ahora bien, ¿tenemos derechos a manipular las cosas más allá de lo que las industrias nos conceden?

Podríamos apelar a alguna clase de derecho de los objetos a ser manipulados desde Bruno Latour y a partir de la confluencia que este pretende en la dicotomía entre las formas de representación (política) y delegación (epistemológica y clasificatoria, confiada a las ciencias y a los tecnócratas). Hay que tener en cuenta que la modernidad ha rechazado sistemáticamente tomar en consideración los derechos de los objetos, por tomar como base de sus desarrollos la oposición sujeto-objeto. Solo si tenemos en cuenta que el modo de clasificación moderno nunca se ha correspondido con lo que realmente sucede en el pensamiento y en la práctica, y aceptamos que no se han reconocido nunca las consecuencias de tales modos de clasificación podemos llegar a reconocer los derechos, la autonomía y la agencia de los objetos. Hay que destacar en este punto la proliferación de híbridos, más allá de las rupturas introducidas por la modernidad que sajan el mundo en dos mitades «a la izquierda el conocimiento de las cosas, a la derecha el interés, el poder y la política de los hombres» (Latour 2007, 17). Los híbridos son objetos que no son naturales y sujetos que no son solo humanos. Hay que redefinir, por tanto, el estatuto de los objetos, su capacidad para expresar una fuerte oposición a nuestra voluntad. Hoy, los objetos están hechos de múltiples conexiones tentaculares, con ligazones arriesgadas; han adquirido la disposición de poner en movimiento consecuencias inesperadas, incluso a largo plazo, y por lo tanto más imprevisibles e incontrolables. Esos objetos no existen de manera separada y por oposición a los sujetos, sino que en ellos los hombres y mujeres estamos implicados. De este modo es como se puede hablar de hibridación inclasificable, de nodos en una red que puede hacer saltar todos los ordenamientos. No obstante, estos híbridos dotados de agencia no son nada nuevos; en el movimiento constructivista ruso, el artista y crítico de arte Borís Arvatov planteaba la idea de una estética pragmática del arte industrial como una forma productiva de afrontar la reutilización del objeto (Kiaer 1997).

Con todo, Arvatov ya valoró que para que los objetos se ensamblen deben ser objetos abiertos, transparentes, que no escondan su código a los usuarios. En la contemporaneidad, dadas las condiciones industriales y el orden económico descrito y aludido, para tener éxito en el concepto de agenciamiento debemos ser desobedientes tecnológicamente, y esto significa ser capaces de transgredir. La cuestión en este punto es la capacidad de cometer infracciones desde el universo zombi; ahora bien, ¿por qué desde ahí?

\section{Transgresión a partir desde el zombie media}

«Los medios zombis son los muertos vivientes de la cultura de masas» (Hertz, Garnet y Parikka, 2011) , aquel hardware y software que han entrado en desuso. Los medios zombis son las máquinas que ya conocemos y no nos duele reventarlas, abrirlas, descomponerlas y diseccionarlas, como argumentaremos después. Son «híbridos» en el sentido que acabamos de exponer, y en muchos casos ya han fallado, nos han ayudado a cobrar conciencia de la realidad, abriéndola a nuevas posibilidades, incitando un imaginario colectivo y a nuevas capacidades de agenciamiento. Son accesibles, los encontramos casi siempre en los cajones de nuestras casas y, en términos generales, han depreciado su condición económica, lo que es especialmente apropiado en situaciones de crisis como la contemporánea, permitiéndonos el ensayo lúdico con ellos sin ninguna clase de temor a su posible destrucción. Nuestra relación con ellos suele presuponer y prefigurar una conducta lúdica creativa que separa el objeto de su uso y su función atribuida, abriéndose en este sentido a propuestas de carácter estético. Podemos convertirlos, por tanto, en cajas transparentes.

\section{Anarqueología de los medios}

Hablamos de transgredir; pero ¿qué hay que transgredir? El hardware que los constituye, el software que los funcionaliza, no ya desde el Do it yourself(DIY), sino hacia un Do it yourself with others (DIYW0), uniendo el parlamento de los sujetos y los objetos, trascendiendo la perspectiva de carácter individualista que se recoge en el primer acrónimo. Solo evidenciando las carencias del modo individual y hegemónico en el trato con la tecnología, y destacando las capacidades de colaboración no solo entre los sujetos, sino también con los objetos, es posible trascender el entorno autocrático al que hemos hecho referencia con anterioridad. Esto supone que hay que transgredir también la teoría en la que se basan. Si los zombie media mantienen su relación con la arqueología de los medios, lo que aquí proponemos es desdibujarla con una anarqueología. Dado que «la arqueología describe discursos como prácticas específicas en el elemento del archivo» (Foucault 1997, 223) y el archivo es «la ley de lo que puede ser dicho, el sistema que rige la aparición de los enunciados como acontecimientos singulares» (Foucault 1997, 219), con frecuencia, 


\section{artnodes}

ambas cosas presuponen un concepto de tiempo lineal, basado en la idea de progreso, a pesar de que pretendan desenmascarar los dispositivos de poder enmarcados en la relación saber-poder. Hay que anotar en este punto, dado que la lectura contradice las críticas de Foucault al pensamiento lineal, que esta es la crítica que elabora Rudi Visker (Visker 1995). Cabe decir que Visker destaca que el objetivo de Foucault es la producción de nuevos sistemas de ordenamiento desentrañados genealógicamente y de manera crítica. Es dicha producción de nuevos órdenes, a juicio de Visker, lo que provoca que queden restos de linealidad en sus teorías, aunque sea una linealidad que descentre los modos de sistematización generados por los dispositivos de producción de verdad. Es esta condición la que convierte Ios archivos en algo muerto, algo digno de ser ubicado y guardado en algún lugar para el estudio de una época. Nosotros entendemos que se puede producir un descentramiento a partir de la idea de anarchivo elaborada por Zielinsky. Se trata de una actitud de investigación y práctica que se mueve con libertad a partir de encuentros de carácter fortuito, en vez de tratar de encontrar la linealidad de las cosas de manera forzada. Más concretamente, Zielinsky, en la entrevista realizada por Moller (Moller 2016), hablará de variantología: «Bajo el concepto más amplio de variantología entiendo la suma imaginaria de todas las posibles genealogías de los fenómenos mediales [...] Los medios no son producto de una tendencia de lo primitivo a lo complejo relacionado con la historia, sino intentos construidos de conectar lo que está separado» (Moller 2016). De este modo, en un anarchivo podemos encontrar todo lo olvidado, las tecnologías ya disfuncionales, las descartadas, las erróneas, todas las que se han perdido. Desde ahí se tratará de llegar a una anarqueología de los medios, una teoría basada más en lo posible que en lo real, en propuestas que establezcan nuevas relaciones de temporalidad entre pasado, presente y futuro, un anarchivo dinámico, operativo y práctico, que sea susceptible de actualizarse constantemente no en relación con lo que las cosas fueron, sino enmarcado en el tiempo de lo verosímil y posible, es decir, de lo que algo puede llegar a ser. Rudi Visker usó hace ya más de una década el término para describir, en relación con la teoría de Foucault, un método que no se somete al paradigma según el cual es necesario identificar el «objeto típico de una experiencia originaria» (Visker 1995, 309). La preeminencia de lo posible frente a lo real implica, necesariamente, una actitud lúdica en la que se trata de modificar la historia para correlacionarla con otros futuros posibles.

Un ejemplo de lo que significa trabajar con anarchivos lo encontramos en la obra Tapebook (Escudero 2014), del artista español César Escudero Andaluz. Este trabajo es un ejercicio de resignificación del concepto de interacción audiovisual. En este caso, el usuario puede escuchar, en cintas analógicas de casete, los perfiles actuales de centenares de autores, críticos, pensadores y colectivos relacionados con el media artpublicados en la red social Facebook. Este juego entre el usuario como mecanismo activo y el dispositivo de registro portable, en peligro de extinción, genera un juego de tiempos anacrónico entre el proceso y la contemplación que produce un desequilibrio entre los propios contenidos que se escuchan y el origen de los mismos. Son dos naturalezas distintas, son un Frankenstein gramatical en términos de media y dispositivo que provoca una práctica, una experiencia de incapacidad y, a la vez, de placer maquínico que descubre el defecto/ efecto de esa relación entre contenido y aparato obsolescente.

La relación anarqueológica necesita de una práctica. En el quinto punto de su manifiesto sobre los zombie media, Jussi Parikka afirma que «es ya tiempo de desarrollar el paso de un método textual a lo material, un material que tenga en cuenta la cultura de la economía política de los medios contemporáneos» (Hertz, Garnet y Parikka, 2011). Esto significa que debemos usar los medios desde un ámbito creativo como es la producción artística. Tenemos que recordar que proponíamos transgredir la teoría, pero también el hardware y el software.

En ese aspecto, el artista estadounidense Cory Arcangel, y en concreto su trabajo Super landscape \#1 (Arcangel, cory arcangel 2005), plantea una relación del uso del medio como método artístico desde la manipulación del software, en concreto, un videojuego clásico como es Mario Bros, seleccionando y aislando los elementos principales del juego más superfluos, las nubes. Estas nubes están prefabricadas, se repiten, son objetos de producción y no participan del juego en el aspecto interactivo, sino que son el recurso gráfico para indicarnos el entorno del juego. Este uso del objeto de producción como elemento destacable se aprecia más claramente, en Cory, en su trabajo de reconfiguración del hardware denominado Self playing Nintendo 64 NBA Courtside 2 (Arcangel 2011). En esta propuesta, Arcangel crea una especie de selfie acción zombi, producto del jaqueado de un dispositivo de juego (Nintendo 64) que genera un sistema cerrado de interacción con los propios contenidos del videojuego, fabricando una sensación de usuario como un objeto del juego a la deriva, el jugador como un caminante muerto. Si bien estas propuestas pueden ayudarnos a ilustrar esa relación con el objeto de juego y de su comportamiento activo o inactivo en un contexto de interacción, es importante apuntar también como ejemplo el cadáver electrónico ReFunt Media \#7 (Galou, Verbruggen y Gieskes 2014), creado por Benjamin Galou, Tom Verbruggen y Gijs Gieskes. Este engendro reciclista se muestra como un enjambre de sistemas tecnológicos que consiguen la resurrección y mantener vivo el propio flujo de la imagen a través de protocolos y tecnologías hardware ensambladas. Esta resurrección genera una reflexión sobre la posibilidad de actividad creativa desde los dispositivos fallecidos; y no solo eso, sino que esa posibilidad de expresión y creatividad se puede apreciar en el intento de revisión de dispositivos agonizantes, como es el caso de The Collapse of PAL (Menkman 2010), de Rosa Menkman, un epílogo electrónico en torno a un tipo de señal codificada videográfica en desuso actualmente. La propia naturaleza de la señal, agonizando, genera belleza. Fabrica un ente atractivo, relacionado con la experiencia abstracta y que nos 


\section{artnodes}

http://artnodes.uoc.edu

Apocalipsis media

permite sentir que esa señal, aun agonizando, con sus carencias sigue viva. Por último, como ejemplo de reordenación del uso, apuntaremos el trabajo del artista sudafricano Rowan Smith 1/2000th if a Supernova (Smith 2009). Esta pieza está formada por un conjunto de flashes de cámara analógica que generan una estrella; mediante un temporizador los flases se activan, generando un nuevo objeto a explorar que dibuja un enjambre de catastróficas instrucciones. Su aspecto ha variado; su capacidad para su uso, no. Sigue manteniendo la sintaxis, pero su semántica ha cambiado.

\section{Disección, regeneración y reparación}

Todo lo que ha quedado expuesto hasta aquí, junto con otros ejemplos ilustrativos aparte de los mencionados, se concreta en tres posibles modos de operar:

1) La disección, que supone la apertura de posibilidades de recombinación de elementos. Este es el caso del trabajo de Evan Roth Internet cache self portrait (Roth 2014), donde su composición de imágenes recopiladas pasivamente a través de la navegación diaria privada en la red genera una estructura mosaico abierta inabarcable que recompone relaciones de significación medial entre privacidad y signos, o el trabajo del artista aragonés Nestor Lizalde Velvet transcode (Lizalde 2010), diseccionando y reconstruyendo de forma digital interactiva audiovisual el disco analógico de rock The Velvet Underground \& Nico.

2) La regeneración, desde la que es posible dar nuevos usos al objeto para hacerlo revivir. En este aspecto, Marinos Koutsomichalis, con su instalación Oiko-nomic Threads (Koustomichalis 2013), crea esa idea de resurrección de los usos mediante el uso de telares.

3) La reparación a partir de la que mejoran o se desvían los usos ya acostumbrados de los dispositivos de referencia de que se trate. Es en este punto donde las arquitecturas de la necesidad y desobediencia electrónica de Ernesto Oroza (Oroza 2014) tienen espacio, esas reparaciones de objetos hechos con Naplam B (gasolina con poliestileno).

La producción creativa elaborada desde la tríada implica hibridar epistemología y política, defendiendo una tecnología democratizada, pero también ecológica y sostenible. Con esto nos referimos, por una parte, a cómo se puede desviar el logos de la tecnología desde una crítica de la razón instrumental, relacionando dicho logos con su significado etimológico griego vinculado a la poética, desde la actitud concreta que hemos desarrollado hasta aquí. Por otra parte, anotamos cómo esta se opone a la obsolescencia programada desde la ecosofía de Guattari y sus tres modalidades respecto al mundo, diseñando una ecología que es medioambiental, social y mental (Guattari 2000).

Esto supone una serie de consecuencias para esos sujetos enredados e hibridados con lo tecnológico, ya que la tendencia es que se produzca un empoderamiento desde la propia precariedad, no ya por la ausencia empobrecida de consumo, sino a partir de las posibilidades de la producción. Si la obsolescencia programada está referida a los sujetos como consumidores, proponemos la ecosofía como modo de empoderamiento de los sujetos de producción, unos sujetos armados de conocimiento de caja transparente que serán más capaces de disponer de su propio tiempo. En una situación en la que el tiempo es declarado como uno de los recursos más importantes para la economía, la técnica y el arte, no debemos fijar tanto la atención en el hecho de si disponemos de mucho o poco tiempo, sino cuestionar quién dispone de nuestro ser temporal y del de los demás y cómo lo hace, articulando formas de producción poiética. Las formas de socialidad introducidas por el referido quehacer del DIWO implican una reapropiación de las tecnologías como práctica social (podemos pensar aquí en el circuit bending 30 en las reuniones de socialización y diversos rituales de las hacking nights. La socialidad es, en este punto, también lúdica.

\section{El tiempo de los zombie media. Conclusiones}

Hemos ido avanzando del consumo y el miedo a la producción y la libertad. El tiempo de esos fallos que relatábamos al principio es un tiempo que deja de ser disfuncional a través de una actitud lúdica y creativa. Si bien los zombie media poseen una capacidad disfuncional que invita a la operatividad, es a través de la misma como podemos aprender de otros medios más veloces. En ambos casos la base es procesual y algorítmica, y el aprendizaje ayuda a asumir que los algoritmos se pueden abrir a posibilidades más alternativas. Si básicamente podemos definir un algoritmo como una secuencia finita de instrucciones paso a paso, las posibilidades creativas y alternativas nos llevan a entenderlos bien, por ejemplo, como «algoritmos», entendiendo una relación fundamental y problemática con la materialidad de la cadencia sonora, provocando una «arritmia» lúdica, como sostiene Miyazaki (Miyazaki 2012), bien, como pretende Galloway, provocando alegoritmos, ya que «jugar un juego significa jugar al código del juego. Ganar significa conocer el sistema. Por lo tanto, interpretar un juego significa interpretar su algoritmo, pero también describir su alegoritmo paralelo» (Galloway 2006, 87).

3. Conjunto de técnicas e interacciones no formales relacionadas con la manipulación y transformación de circuitos electrónicos estándar desde un carácter experimental, creativo y estético vinculadas a la filosofía DIY (Do it yourself) y la artesanía electrónica. Como fuentes de referencia, apuntaríamos el texto de Reed Ghazala Circuit-Bending: Build Your Own Alien Instruments, así como también Handmade Electronic Music. The Art of hardware hacking, de Nicolas Collins. 


\section{artnodes}

Con todo, Ios zombie media muestran el conflicto entre el modelo de usuario impuesto por las grandes compañías de fabricación de tecnología y la afirmación del postulado del comportamiento real de aquellos usuarios que perturban una visión concreta de los usos de la tecnología (destacamos aquí el término postulado, ya que estas líneas no están destinadas específicamente al estudio del referido comportamiento real); produce jugadores no neutrales que asumen una postura crítica sobre el mundo y los valores del juego, lleva a la adquisición de una conciencia inmersiva a partir de la emersión. Los medios zombis no son inmersivos; por ello favorecen y entrenan una conciencia analítica y con capacidad de evaluación de contenidos frente a la instantaneidad de los medios contemporáneos.

\section{Epílogo}

Pasados los treinta días que establece el Reglamento del Senado en su artículo 169-1, el Gobierno español matizó su respuesta al senador Mulet, en relación con los protocolos establecidos en la legislación referentes a un apocalipsis zombi, y lo hizo en relación con el significado del término zombi según la RAE. Para su primera acepción (persona que se supone muerta y reanimada por el arte de la brujería con el fin de dominar su voluntad), el Gobierno «no había adoptado ningún protocolo específico dada la dudosa probabilidad de que se produzca semejante circunstancia bajo tales premisas» (El Hufftington Post 2017). En relación con la segunda de las acepciones del término (atontado, que se comporta como un autómata), el Ejecutivo valoró que, a pesar de poseer diversas líneas de actuación, dudaba de que quienes pudieran ser calificados como tales llegaran a protagonizar una situación de apocalipsis. Al Gobierno no le faltaba cierto grado de razón respecto al inmovilismo, pero, aunque proliferan los zombis en estos tiempos, al menos los que son producto de actuaciones que acrecientan la ausencia de conocimiento, la manipulación mediática, la ausencia de unos usos verdaderamente democráticos de la técnica y cierta incapacidad de transgresión, eso no significa que no sea posible un cambio radical por una revitalización lúdica de la curiosidad y la desobediencia. No sabemos si desde aquí se propiciará o no un apocalipsis, pero al menos sí que es importante conocer los códigos de un posible juego para la resurrección electrónica.

\section{Referencias bibliográficas}

Arcangel, Cory. 2005. Cory arcangel. [Fecha de consulta: 02/02/2018] http://www.coryarcangel.com/things-i-made/2005-021-superlandscape-1/.

Arcangel, Cory .2011. Coryarcangel.com. [Fecha de consulta: 03/02/2018]. http://www.coryarcangel.com/things-i-made/2011115-self-playing-nintendo-64-nba-courtside-2.
Barron, Melissa. 2010. Melissa Barron. [Fecha de consulta: 12/12/2017]. http://melissabarron.net/screencaptures.html.

Beck, Ulrich. 2002. La sociedad del riesgo global. Madrid: Siglo XXI.

Castells, Manuel. 2006. La era de la información (Vol. 3): Economía, sociedad y cultura. Fin de milenio. Madrid: Alianza.

Collins, Nicolas. 2009. Handmade Electronic Music: The Art of Hardware Hacking. Londres: Routledge.

Deleuze, Gilles. 1999. Conversaciones. 1972-1990. Valencia: PreTextos.

El Hufftington Post, redacción. 2017. «La loca respuesta del gobierno ante la pregunta de un senador sobre el apocalipsis zombi.» El Hufftington Post.

Escudero, César. 2014. Escudero andaluz. [Fecha de consulta: 22/12/2017]. http://escuderoandaluz.com/2014/01/28/tapebook/.

Foucault, Michel. 1997. La arqueología del saber. México: Siglo xxl.

Galloway, Alexander R. 2006. Gaming. Essays o Algorithmic Culture. Minneapolis: University of Minnesota Press.

Galou, Benjamin; Tom Verbruggen y Gijs Gieskes. 2014. recyclism. com. [Fecha de consulta: 01/01/2018]. http://www.recyclism. com/refunctmedia_v5.php.

Ghazala, Reed. 2005, Circuit-Bending: Build Your Own Alien Instruments. New. [Fecha de consulta: 02/02/2018]. Jersey: Wiley.

Gil Calvo, Enrique. 2003. El miedo es el mensaje. Riesgo, incertidumbre y medios de comunicación. Madrid: Alianza.

Guattari, Feliz. 2000. Las tres ecologías. Valencia: Pre-Textos.

Hertz, Garnet y Jussi Parikka. 2011. Five Principles of Zombie Media. [Fecha de consulta: 10/10/2017]. https://www.researchgate.net/ publication/273062231_Five_Principles_of_Zombie_Media.

Kiaer, C. 1997. «Boris Arvatov's Socialist Objetcs». October(MIT Press) 81: 105-118.

Koster, Raph. 2000. Raph Koster's Website. 8. [Fecha de consulta: 29/09/2017]. https://www.raphkoster.com/games/essays/ declaring-the-rights-of-players.

Koustomichalis, Marinos. 2013. Marinos Koustomichalis. [Fecha de consulta: 02/02/2018]. http://marinoskoutsomichalis.com/ oikonomic-threads/.

Latour, Bruno. 2001. La esperanza de Pandora. Ensayos sobre la realidad de los estudios de la ciencia. Barcelona: Gedisa.

Latour, Bruno. 2007. Nunca fuimos modernos. Ensayo de antropología simétrica. Buenos Aires: Gedisa.

Lizalde, Nestor. 2010. Nestor Lizalde. Nuevos Medios. [Fecha de consulta: 07/01/2018]. http://www.nestorlizalde.com/index.php?/ works/velvet-transcode/.

Menkman, Rosa. 2010. Vimeo. [Fecha de consulta: 03/02/2018]. http://vimeo.com/12199201.

Miyazaki, Shintaro. 2012. «Computational Culture. A journal of software studios». Algorhythmics: understanding micro-temporality in computational cultures. [Fecha de consulta: 26/05/2017]. 


\title{
artnodes
}

http://artnodes.uoc.edu

Apocalipsis media

http://www.computationalculture.net/algorhythmics-understanding-micro-temporality-in-computational-cultures.

Moller, Nathalia. 2016. «La Fuga, 14.» Entrevista con Siegfried Zielinski. [Fecha de consulta: 10/02/2018]. http://2016.lafuga.cl/ entrevista-con-siegfried-zielinski/531.

Oroza, Ernesto. 2014. Ernesto Oroza. Architecture of necessity, Technological Disobedience, Moral Modulor, Moire house, Objects of Necessity, Generic objects, Potential house.... [Fecha de consulta: 31/02/2017]. http://www.ernestooroza.com/objects-of-naplam-b.
Pozzi, Sandro. 2010. «Las máquinas se apoderan de Wall Street y provocan el pánico en el mercado.» El País, 7 de mayo.

Roth, Ewan. 2014. Ewan Roth.[Fecha de consulta: 02/02/2018]. http:// www.evan-roth.com/work/internet-cache-self-portrait/.

Smith, Rowan. 2009. Rowan Smith. [Fecha de consulta: 28/01/2018]. http://www.whatiftheworld.com/publication/rowan-smith/.

Visker, Rudi. 1995. Foucault. Genealogy as Critique. Londres: Verso.

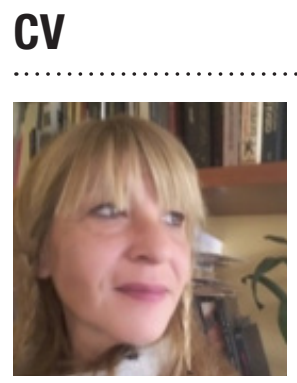

\author{
Marina Pastor \\ Universitat Politècnica de València \\ mpastor@esc.upv.es \\ Facultat de Belles Arts \\ Universitat Politècnica de València \\ Camí de Vera, s/n \\ 46022 València \\ València \\ Espanya
}

Licenciada en Filosofía y Ciencias de la Educación, doctora en Bellas Artes por la UPV.

Actualmente es profesora del Departamento de Escultura de la Universidad Politécnica de Valencia y del máster de Artes Visuales y Multimedia. Ha sido directora del máster de Producción Artística (UPV), del posgrado en Arte, Producción e Investigación de la Facultad de Bellas Artes y del Departamento de Escultura. Miembro desde 1998 del grupo de Investigación I+D+I Laboratorio de Creaciones Intermedia.

Ha participado en varios proyectos de investigación financiados, entre los que se encuentran «Reconstrucción de obras artístico-sonoras pertenecientes a la vanguardia histórica europea (1913-1945)»0 «Percepción ampliada, un sistema dual de realidad aumentada». Desde 1999 su trabajo se centra en la relación entre arte y nuevas tecnologías; en torno a ello ha publicado textos como «La imagen crítica», en el libro Radicantes. Danza y otras especies (IVAM, Valencia, 2018); La construcción difusa del paisaje. Latitud norte e imaginarios colectivos. Vínculos Nórdicos. Ética y estética del habitar (Valencia, 2013) 0 Ruidos y susurros de las vanguardias (1909-1945) (Valencia, 2008); «De guillotinas... y otros modos de decapitación», en Del mono azul al cuello blanco. Transformación social y práctica artística en la Era Postindustrial (Valencia, 2003), y Representaciones de la sexualidad en las artes. Miradas sobre la sexualidad en el arte y la literatura del siglo xx en Francia y España (2001). Ha publicado diferentes artículos de investigación en revistas, como «Nación -frontera. Apuntes sobre la expansión de la frontera y algunas de sus implicaciones» (Murcia, 


\section{artnodes}

http://artnodes.uoc.edu

Apocalipsis media

2017), «La semiótica del alien» (Unstate, Valencia, 2017), «Noise into play. Disonancias e indeterminación de lo sonoro en lo lúdico» (Ausart, 2015) 0 «Acontecimientos, torpezas y derivas: generadores ludocráticos» (Ausart, 2014). Ha participado en exposiciones como femCode $<$ Arte+Mujeres+Tecnología $>$ (Valencia, 2014), Soirée Sonore (Le Gresas. Chalet en Bois Rond. Montebello. Canadá, 2007); Festival La Flexió Verbal (Alicante, 2006); No somos idéntic@s (Valencia, 2006). International Digital Art (Mylos Cultural Center Tessalonica, 2005); Reinventando la radio. Exploradores sonoros (Radio Educación, México, 2004).

Ha participado como ponente en congresos como «Interface Politics» (Barcelona, 2016), «Arte y políticas de identidad» (Murcia, 2017) 0 «I Fòrum Internacional Una (altra) Història de l'art. Dones i Art» (Valencia, 2018), y comisariado exposiciones como 4 Biennial European Academies of visual Arts (Maastricht, 1993) y Art Public. IX Mostra d'art públic per a joves creadors (Valencia, 2006).

\section{CV}

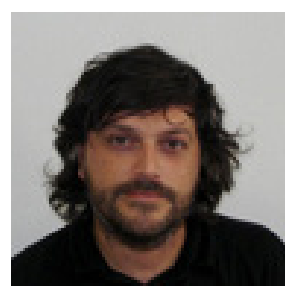

\section{Moisés Mañas}

Universitat Politècnica de València

moimacar@esc.upv.es

www.moisesmanas.com

https://upv-es.academia.edu/moisesmanas

Facultat de Belles Arts

Universitat Politècnica de València

Camí de Vera, s/n

46022 València

València

Espanya

Licenciado en Bellas Artes, doctor en Artes Visuales e Intermedia por la UPV y posgrado en Programación de Intranets e Internet. CFP-Dpto. de Informática de Sistemas y Computación. DISCA.UPV.

Actualmente, es profesor de Media Art en el Departamento de Escultura de la Universidad Politécnica de Valencia. Director desde el año 2012 del máster oficial de Artes Visuales y Multimedia (UPV). Miembro desde 2000 del grupo de investigación I+D+I Laboratorio de Luz.

Ha presentado comunicaciones y formado parte de mesas redondas en festivales de arte electrónico como FutureEverything (Manchester), Ars Electrónica-Electro Campus (Linz-Austria), Transmediale (Berlín), AMAZE (Berlin) e ISEA (Estambul).

Desde 1996 hace uso de las nuevas tecnologías aplicadas al arte. Su trabajo ha sido presentado y premiado en diferentes festivales y exposiciones relacionados con arte y tecnología, dentro y fuera de 


\section{artnodes}

http://artnodes.uoc.edu

Apocalipsis media

España desde 1997. Cabe destacar Parlamento1\#, en el IVAM-Institut Valencià d'Art Modern, 2017, Los algoritmos suaves (2018), Reinventar 10 posible-Accionar lo imaginable (CCCC, Centro del Carmen, Centro de Cultura Contemporánea, Valencia, 2017), Remote Signals (Tallin, Estonia, 2016), Media Art Futures Festival (Murcia, 2015), el Premio Internacional de Arte Electrónico BEEP-ARCO 2013 (mención especial del jurado), Technical Unconscious (Oporto, Portugal, 2014), QUARTEL (Abrantes, Portugal, 2014), The WRONG, New Digital Art Biennale (2013), Common Ground (Miami, Estados Unidos, 2012), Museo de Arte Moderno de Santo Domingo (República Dominicana, 2011), Instituto de Desarrollo de las Artes Visuales de La Habana (Cuba, 2011), Galeria Space (Pittsburgh, Estados Unidos, 2011), Spazio Museale di Palazzo Tornielli (Ameno, Italia, 2011), Festival Digital Image-Espacio ENTER (Canarias, 2009), El discreto encanto de la tecnología, Neue Galerie, en Graz (Austria, 2009), MEIAC-Badajoz (2008), ZKM-Center for Arts and Media, Karlsruhe, Alemania (2008-2009), 4. ${ }^{\circ}$ Festival de las Artes de Castilla y León (Salamanca, 2008), Bienal de Valencia (2007), File 04 (São Paulo, Brasil), Mediaterra (2000 y 2004, Atenas, Grecia), Inmedia (2000, Míchigan, Estados Unidos), Observatori-2000, INTERTECNO (2000), Valencia, Festival de Creación Audiovisual de Navarra 98/99/00, Ciber@rt 1999, Muestra itinerante de Cd-Rom españoles (Costa Rica, Santo Domingo, Argentina, Colombia), Museo Reina Sofía 1999/00, II Muestra Internacional de Arte en CDROM, MECAD (Sabadell, Barcelona, 2000). Vértigos: Artes Visuales. Online/Offline (Madrid, 2000), «Entre les yeux et ce qu'ils voient» (Lieja, Bélgica, 2001). Pasos de Cebra (Injuve, Madrid, 2001), Situaciones 2001, Arte Joven, Injuve 2000 (itinerante en Uruguay, Argentina y Perú), «Front», Edgard Neville (Alfafar, 2003), Lenguajes Híbridos (2003, Gandía), Robotic Art, Interactive Instalations \& Robots (Pittsburgh, Estados Unidos, 1999), Analoguic Bytes (Southampton, Reino Unido, 1997). 